\title{
Lattice dynamics of antimony under pressure
}

\section{A Minelli ${ }^{1}$, S Souliou ${ }^{2}$, A Romero $^{3}$, V Dmitriev $^{4}$, A Bosak $^{5}$ \\ ${ }^{1}$ University of Oxford, Inorganic Chemistry Laboratory, Oxford, UK, ${ }^{2}$ Institut for solid state physics, Karlsruhe institute of technology, Germany, ${ }^{3}$ Department of Physics and Astronomy, West Virginia University, WV, ${ }^{4}$ European Synchrotron Radiation Facility (ESRF), Grenoble, France, ${ }^{5}$ European Synchrotron Radiation Facikity (ESRF), Grenoble, France Arianna.minelli@chem.ox.ac.uk}

The group Va elements, with the only exception of nitrogen, crystallise in the rhombohedral A7 structure. It comes from a small distortion with respect to a simple cube. The distorted structure leads to twice as many atoms in the primitive cell, inducing a gain in the electron energy with a connected gap opening, called a Peierls distorted structure. Under pressure, the distortion is reduced, but remains finite, as antimony transforms through a series of highly complex structure, before adopting as last the BCC (body-centred cubic) phase. Through the combination of diffuse and inelastic x-ray scattering compared with ab-initio calculation and a symmetry-based phenomenological model of the phase transitions, the dynamics behaviour approaching the transition is analysed. The main diffuse scattering features and, to some extent, the peculiarities in the lattice dynamics of the A7 phase will be explained by the instability of the primitive cubic network with respect to a correlated displacement.

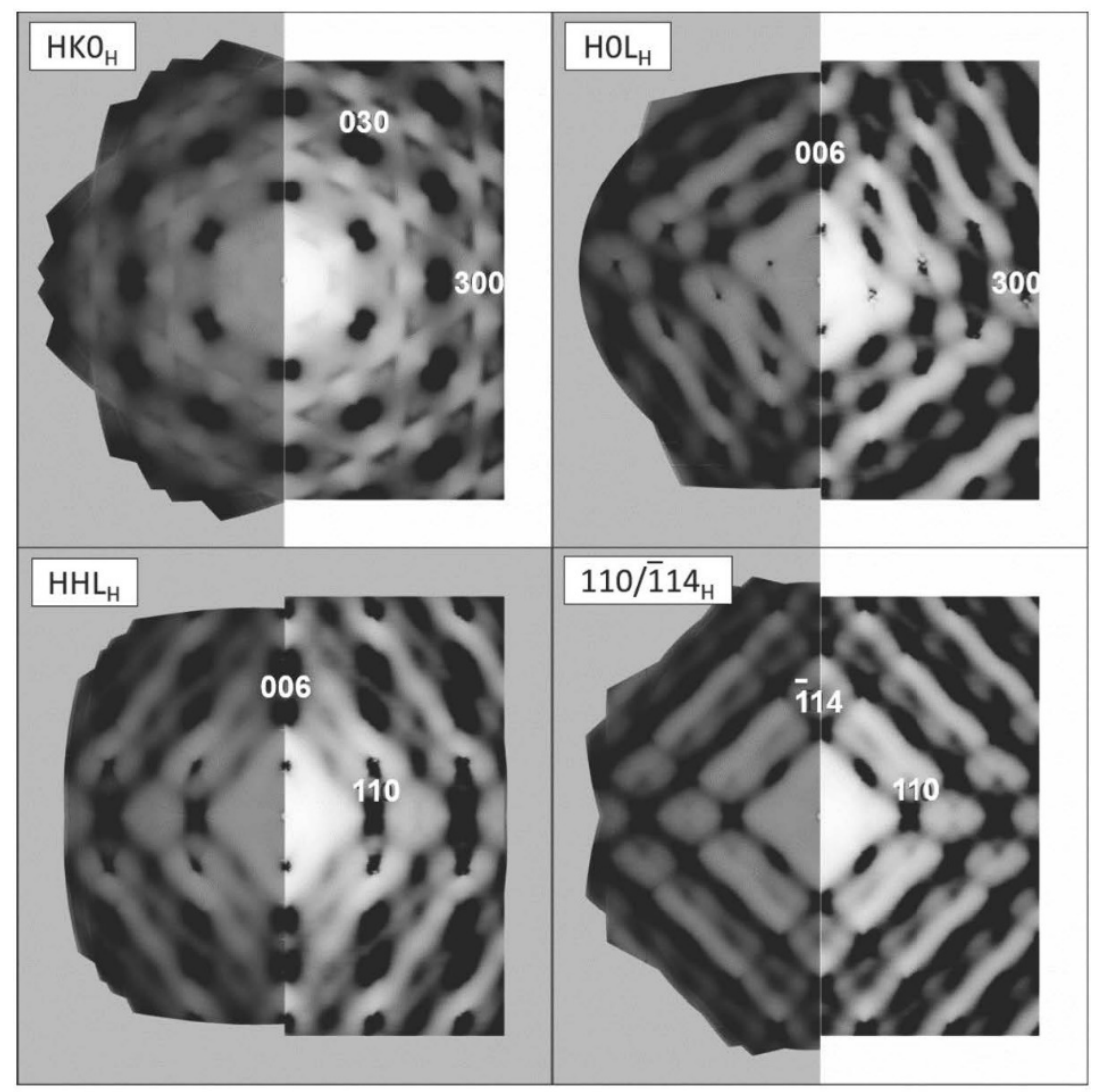

Figure 1.

Acta Cryst. (2020). A76, a227 